\title{
Double versus single homologous intrauterine insemination for male factor infertility: a systematic review and meta-analysis
}

\author{
Apostolos Zavos ${ }^{1}$, Alexandros Daponte ${ }^{1}$, Antonios Garas ${ }^{1}$, Christina Verykouki ${ }^{1}$, Evangelos Papanikolaou ${ }^{2}$, \\ Georgios Anifandis ${ }^{1}$ and Nikolaos P Polyzos ${ }^{3}$
}

\begin{abstract}
Male factor infertility affects $30 \%-50 \%$ of infertile couples worldwide, and there is an increasing interest in the optimal management of these patients. In studies comparing double and single intrauterine insemination (IUI), a trend towards higher pregnancy rates in couples with male factor infertility was observed. Therefore, we set out to perform a meta-analysis to examine the superiority of double versus single IUI with the male partner's sperm in couples with male factor infertility. An odds ratio (OR) of $95 \%$ confidence intervals (CIs) was calculated for the pregnancy rate. Outcomes were analysed by using the Mantel-Haesel or DerSimonian-Laird model according to the heterogeneity of the results. Overall, five trials involving $1125 \mathrm{IUI}$ cycles were included in the meta-analysis. There was a two-fold increase in pregnancies after a cycle with a double IUI compared with a cycle with a single IUI (OR: $2.0 ; 95 \% \mathrm{Cl}: 1.07-3.75$; $P<0.03$ ). Nevertheless, this result was mainly attributed to the presence of a large trial that weighted as almost $50 \%$ in the overall analysis. Sensitivity analysis, excluding this large trial, revealed only a trend towards higher pregnancy rates among double IUI cycles (OR: $1.58 ; 95 \% \mathrm{Cl}: 0.59-4.21)$, but without statistical significance $(P=0.20)$. Our systematic review highlights that the available evidence regarding the use of double IUI in couples with male factor infertility is fragmentary and weak. Although there may be a trend towards higher pregnancy rates when the number of IUls per cycle is increased, further large and well-designed randomized trials are needed to provide solid evidence to guide current clinical practice.
\end{abstract}

Asian Journal of Andrology (2013) 15, 533-538; doi:10.1038/aja.2013.4; published online 27 May 2013

Keywords: homologous; intrauterine insemination; male infertility; meta-analysis; systematic review

\section{INTRODUCTION}

Male factor infertility affects almost $30 \%-50 \%$ of infertile couples worldwide, ${ }^{1}$ with most infertile men experiencing low sperm density or other semen abnormalities without the presence of any specific underlying cause. In these men with idiopathic oligospermia, asthenospermia or teratospermia, although medical treatment may improve semen quality parameters, it is unclear whether such a clinical practice may indeed improve fertility in general. ${ }^{2}$ Taking into account that the possibility of spontaneous pregnancy is $2 \%$ without any therapy, ${ }^{3}$ assisted reproduction is the next logical step to increase the possibility of pregnancy. ${ }^{4}$

Although the selection of the most suitable assisted reproduction protocol for these couples is based on many parameters and differs among assisted reproduction centres, this selection depends on the success rate and the cost of each method. In vitro fertilisation (IVF) with intracytoplasmic sperm injection may result in a live birth rate of about $40 \%$ after successful egg retrieval from couples with male factor infertility and a maternal age $<35$ years. ${ }^{5}$ Nonetheless, the more realistic first-line approach of intrauterine insemination (IUI) is proposed when at least one million normal spermatozoa are present, ${ }^{4}$ given that this is a more cost-effective method that offers the same likelihood of successful pregnancy as IVF. ${ }^{6}$

Many IUI techniques have been used to achieve higher pregnancy rates. Several trials have been conducted testing the effect of two IUIs versus one IUI. The pregnancy rates of women who received two IUIs per cycle have not proven to be superior; ${ }^{7}$ however, in some of these trials, there has been a trend towards higher pregnancy rates in couples with male factor infertility. ${ }^{8}$ We therefore set out to perform a metaanalysis to investigate the effect of double versus single IUI with the male partner's sperm in couples with male factor infertility.

\section{MATERIALS AND METHODS}

Search strategy

Two independent investigators (AZ and NPP) searched PubMed, the Cochrane Library and the ICI without language and year restrictions by using the search terms (intrauterine insemination OR insemination OR IUI) AND (clinical trial OR randomized controlled trial OR double-blind OR single-blind OR random OR randomized). In addition,

${ }^{1}$ Obstetrics and Gynecology, University Hospital of Larissa, Larissa 41110, Greece; ${ }^{2}$ Aristotle University of Thessaloniki, Assisted Reproduction Unit, 1st OB-GYN Department, Thessaloniki 56429, Greece and ${ }^{3}$ University Hospital, Dutch-Speaking Free University of Brussels, Brussels City 1090, Belgium Correspondence: Dr NP Polyzos (n.polyzos@gmail.com)

Received: 18 November 2012; Revised: 2 January 2013; Accepted: 1 February 2013; Published online: 27 May 2013 
we tried to identify any previous systematic reviews of randomized trials in this field. Whenever we came across a previous systematic review, we searched the references for potentially eligible trials. Finally, the references of all eligible trials were also scrutinized and cross-searches were performed in PubMed using the names of lead authors in at least one eligible trial. In addition, the abstract books of the annual meetings of the ESHRE and ASRM were hand-searched for potentially eligible articles.

\section{Eligibility criteria}

We considered eligible, all randomized controlled studies comparing one IUI versus two IUIs per cycle only in couples with male factor infertility. If trials involved patients with several types of infertility that included male factor infertility, we focused only on the eligible patients' subgroup. The trials were considered eligible whenever we were able to extract data regarding the clinical pregnancy or live birth rate for patients with male factor infertility. When separate data were not provided for couples with male factor infertility, the trial was disregarded.

Additionally, we focused only on trials in which patients received the second IUI $48 \mathrm{~h}$ prior to hCG administration. This strategy was adopted because a previous randomized controlled trial showed that when the second IUI in a double IUI cycle is performed later in the postovulatory period (more than $48 \mathrm{~h}$ before hCG administration), no clear benefit in clinical pregnancy is achieved compared with a single IUI. ${ }^{9}$ Consequently, arms or trials in which the double IUI cycle involved one IUI during the periovulatory period and one during the postovulatory period were disregarded from the analysis.

To minimize heterogeneity, we only included trials in which patients received homologous IUI (partners' sperm). Trials with patients undergoing artificial insemination with the donor's sperm were excluded from our analysis. If trials included both patients undergoing IUI with donor or partner sperm, we focused only on the eligible subgroup. The trial was considered ineligible whenever data could not be retrieved.

Crossover trials, in which patients were allocated to one treatment arm in the first treatment cycle and consequently received the alternate treatment in future cycles, were considered eligible only when data regarding pregnancies were available from the first treatment cycle (prior to crossover). Only these data were recorded and analysed.

Finally, trials were considered eligible regardless of the ovarian stimulation regimen used (e.g., gonadotrophins, clomiphene citrate) if these treatments did not differ systematically between the investigated arms. Whenever regiments differed systematically between the compared arms, the trial was not included in the analysis in order to avoid bias.

\section{Data extraction}

Data were extracted by two independent investigators (NPP and AZ). For each eligible trial, we recorded the following items for both arms: authors' names, journal and year of publication, country of origin, number of patients randomized and eligible per arm, number of cycles per arm, type of controlled ovarian hyperstimulation used, mean age at enrolment and severity of male factor infertility. Additionally, the timing of single and double inseminations per cycle and the total number of spermatozoa inseminated in investigational arms were recorded.

The effect of a double IUI compared with a single IUI was calculated by recording the clinical pregnancy rates per cycle for two versus one IUI in couples with male factor infertility.
Trial quality was assessed by using the Cochrane's risk of bias tool. Two independent investigators, AZ and NPP, assessed the quality of trials, and consensus was reached through discussion.

\section{Analysis}

We addressed the odds ratio for the clinical pregnancy or live birth rate through the comparison of double versus single IUI in couples with male factor infertility. For each eligible study group, we estimated the $95 \%$ confidence interval (CI) and the odds ratio (OR) for clinical pregnancy between the groups being compared. Between-study heterogeneity for the odds ratio was evaluated using the $\mathrm{Q}$ statistic. ${ }^{10,11}$ Both fixed effects (Mantel-Haenszel) and random effects (DerSimonian-Laird) modelling ${ }^{11}$ were used for the synthesis of data across studies. When between-study heterogeneity was not observed, the fixed and random effect estimates coincide. In the presence of between-study heterogeneity, random effects provide wider confidence intervals. ${ }^{10}$ Analyses were performed using Revman 5 statistical software. All $P$ values were two-tailed.

\section{RESULTS}

Eligible trials

The electronic search up to October 2012 yielded 2904 items: 1440 from PubMed, 932 from ICI and 532 from the Cochrane Library. The full text of 39 reports was scrutinized; 25 of these were excluded, and 2 additional trials were retrieved from manually searching the reference lists, as well as ASRM and ESHRE abstract books. Sixteen randomized trials were considered potentially eligible and met our inclusion criteria. However, 11 of them were disqualified, eight due to lack of separate data for couples with male infertility and three because they only included women with unexplained infertility (Figure 1). The

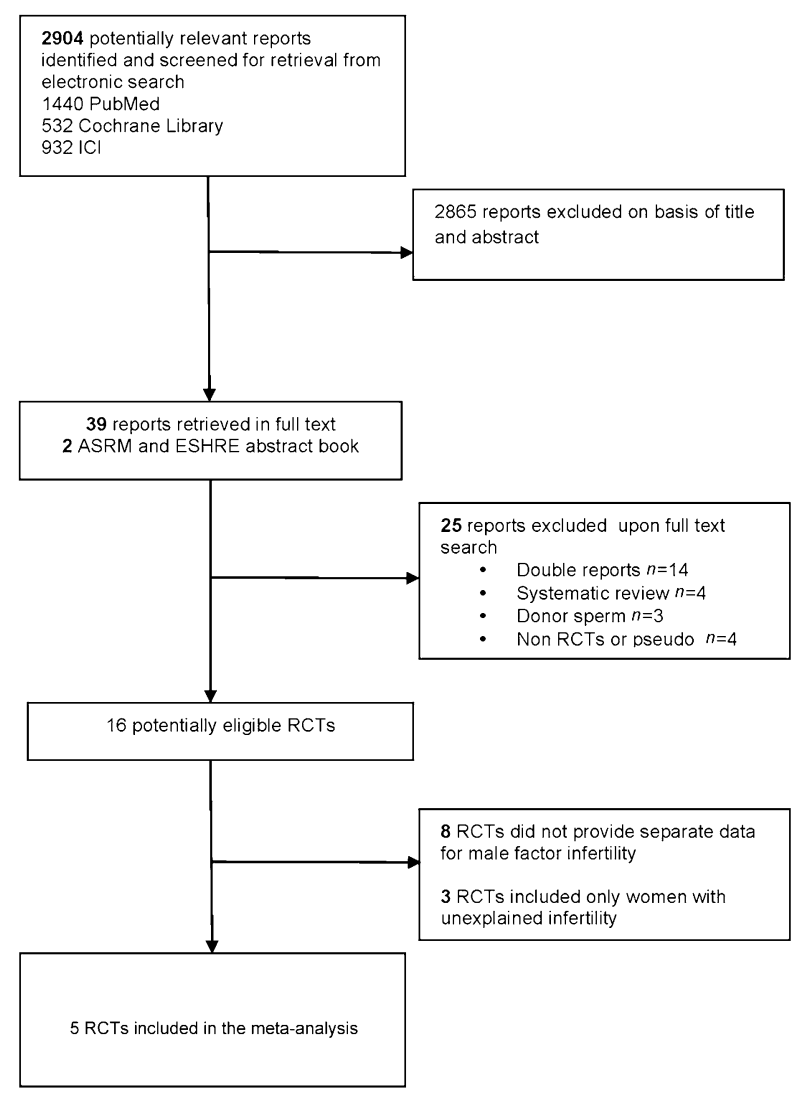

Figure 1 Flowchart diagram of trials selection. 


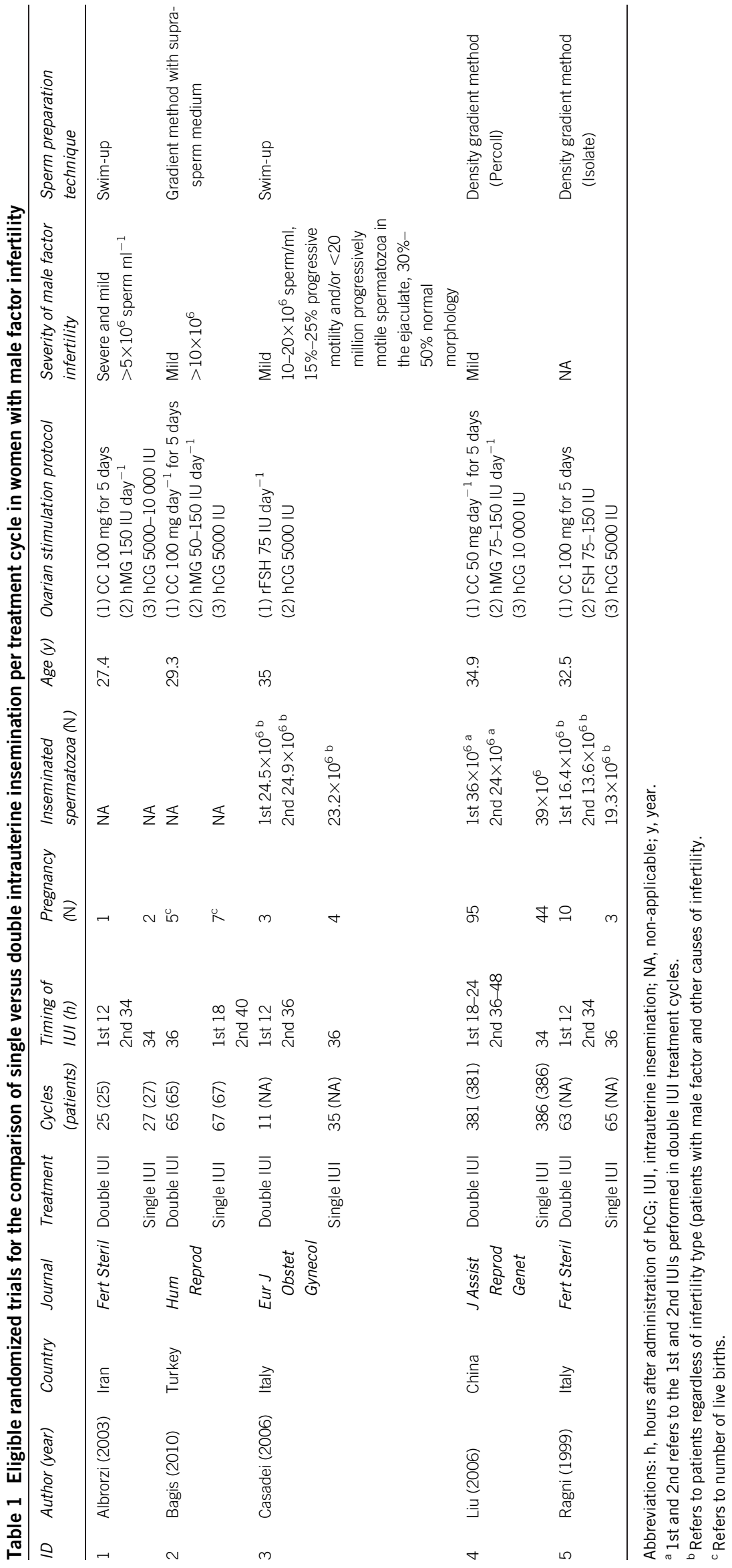




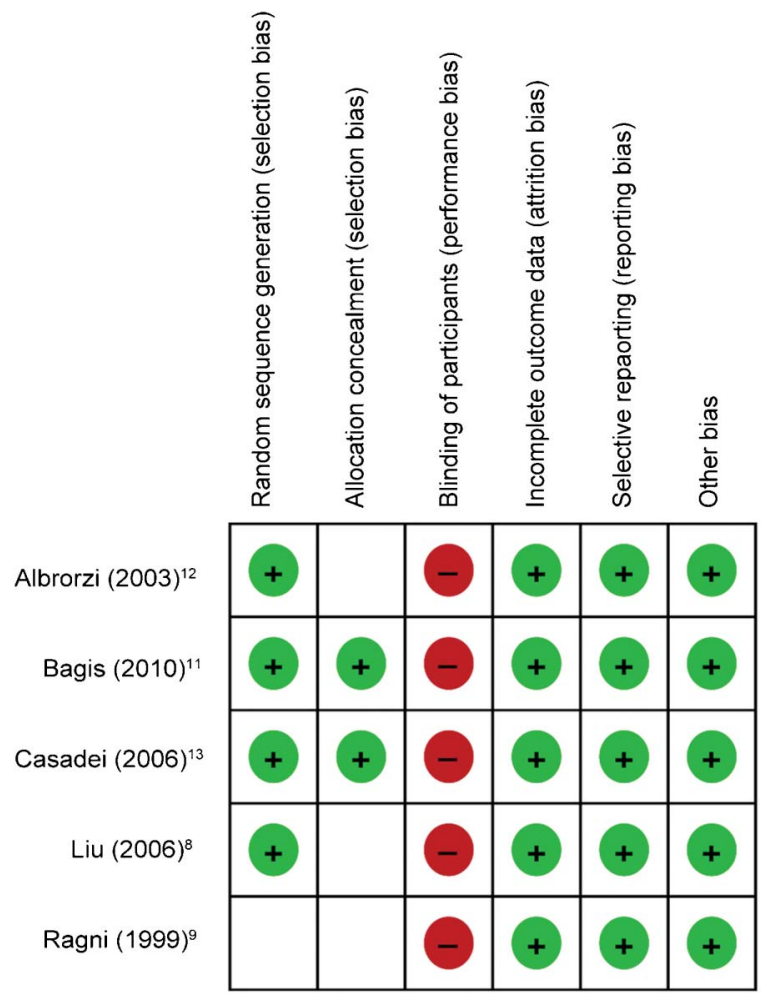

Figure 2 Methodology quality of trials assessed by Cochrane's risk of bias tool.

pregnancy rate was reported as clinical pregnancy in four studies $^{8,9,12,13}$ and as live birth rate in one study. ${ }^{14}$

Finally, five randomized trials published between 1999 and 2011 that involved 1125 treatment cycles were analysed (Table 1). The mean age of women enrolled ranged from 27.4 to 35 years.

Ovulation triggering and ovarian stimulation protocols did not differ among eligible trials. In all trials, ovulation triggering was achieved by the intramuscular injection of hCG with doses of $5000 \mathrm{IU}^{9,13,14}$ or 10000 IU. ${ }^{8,12}$ Most of the trials used the combination of clomiphene and gonadotrophins to induce ovulation..$^{8,9,12,14}$ Only one trial with ovarian stimulation involved only the use of gonadotrophins. ${ }^{13}$

The timing of IUI was also comparable in all of the eligible trials. The single IUI per treatment cycle was performed around the time of ovulation ( $\sim 36 \mathrm{~h}$ after hCG administration), whereas in double IUI cycles, the first IUI was performed in the preovulatory period and the second in the periovulatory period.

The severity of male factor infertility differed among the eligible trials. Two of the trials included only patients with mild male factor infertility, ${ }^{8,13,14}$ whereas one trial included patients with mild and severe male factor infertility. ${ }^{12}$ One trial did not specify the severity of male factor infertility among couples included. ${ }^{9}$

Two different sperm preparation techniques were used in the eligible trials. Half of the trials used the density gradient technique, ${ }^{8,9,14}$ whereas the other two trials ${ }^{12,13}$ used the swim-up technique.

Finally, the number of spermatozoa inseminated was reported in three trials. In all trials, the cumulative number of spermatozoa inseminated was higher in the double IUI arms.

\section{Quality assessment of trials}

The quality of the eligible trials was assessed using Cochrane's risk of bias tool. Two independent investigators separately assessed the risk of bias of each individual trial, and a consensus was reached after discussion. Although several methodological parameters of the trials were satisfactory, with four out of five trials ${ }^{8,12-14}$ providing a sufficient randomisation mode and two ensuring allocation concealment, ${ }^{13,14}$ none of the studies was blinded (Figure 2). One trial ${ }^{12}$ was a crossover trial; however, data regarding pregnancies were available from the first treatment cycle (prior to crossover), and the trial was included in the analysis.

\section{Meta-analysis}

Five randomized controlled trials addressed the comparison of double and single IUI for male factor infertility; these involved a total of 545 cycles with double IUI and 580 cycles with single IUI. After double IUI, there were 114 (20.9\%) pregnancies. After single IUI, 60 (11.0\%) pregnancies were recorded. According to the random effects model, there was a twofold increase in pregnancies after a cycle with double IUI compared with a cycle with single IUI (OR: $2.00 ; 95 \%$ CI: $1.07-3.75 ; P<0.03$ ) (Figure 3). There was no between-study heterogeneity $\left(I^{2}=34 \%\right)$, and therefore, the fixed effects model yielded a similar result (OR: $2.32,95 \%$ CI: $1.65-3.26, P<0.001)$. Because one trial ${ }^{8}$ was weighted at almost $50 \%$ when using the random effects model and made up approximately $74 \%$ of the results when using the random effects model, a sensitivity analysis was performed that excluded this trial. Although there was a trend towards double IUI cycles (OR: 1.58; 95\% CI: 0.59-4.21), there was no significant difference between the two arms $(P=0.20)$.

\section{DISCUSSION}

Our systematic review highlights that the available evidence regarding the use of double IUI among couples with male factor infertility is fragmentary and weak. Although the results show an overall increase in pregnancy rates among couples with male factor infertility who were treated with two IUIs per treatment cycle, this is mainly attributable to a single trial with a high risk of bias. ${ }^{8} \mathrm{~A}$ sensitivity analysis was performed excluding this trial, and the results bring into question

\begin{tabular}{|c|c|c|c|c|c|c|c|c|c|}
\hline \multirow{2}{*}{$\begin{array}{l}\text { Study or Subgroup } \\
\text { Ragni }(1999)^{9}\end{array}$} & \multicolumn{2}{|c|}{ Single IUI } & \multicolumn{2}{|c|}{ Double IUI } & Weight & $\begin{array}{l}\text { Odds Ratio } \\
\text { M-H, Random, } 95 \% \mathrm{Cl}\end{array}$ & \multicolumn{2}{|c|}{$\begin{array}{c}\text { Odds Ratio } \\
\text { M-H, Random, } 95 \% \text { Cl }\end{array}$} & \\
\hline & 10 & 63 & 3 & 65 & $16.0 \%$ & $3.90[1.02,14.91]$ & & 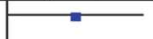 & \\
\hline Albrorzi $(2003)^{12}$ & 1 & 25 & 2 & 27 & $5.9 \%$ & $0.52[0.04,6.13]$ & & & \\
\hline Liu $(2006)^{8}$ & 95 & 381 & 44 & 386 & $48.2 \%$ & $2.58[1.75,3.81]$ & & & \\
\hline Casadei $(2006)^{13}$ & 3 & 11 & 4 & 35 & $11.3 \%$ & $2.91[0.54,15.70]$ & & & \\
\hline Bagis $(2010)^{14}$ & 5 & 65 & 7 & 67 & $18.7 \%$ & $0.71[0.21,2.38]$ & . & & \\
\hline Total $(95 \% \mathrm{Cl})$ & & 545 & & 580 & $100.0 \%$ & $2.00[1.07,3.75]$ & & & \\
\hline Total events & 114 & & 60 & & & & & & \\
\hline $\begin{array}{l}\text { Heterogeneity: } \mathrm{Tau}^{2}= \\
\text { Test for overall effect }\end{array}$ & $\begin{array}{l}17 ; \mathrm{Chi}^{2} \\
=2.16(f\end{array}$ & $\begin{array}{l}=6.03 \\
P=0.0\end{array}$ & df $=4(F$ & $=0.20$ & $; l^{2}=34 \%$ & & $\begin{array}{ll}0.01 & 0.1 \\
\text { Favours } & \text { Single IUI }\end{array}$ & $\begin{array}{l}10 \\
\text { Favours Do }\end{array}$ & $\begin{array}{ll}100 \\
\text { le IUI }\end{array}$ \\
\hline
\end{tabular}

Figure 3 Meta-analysis plot for the comparison of double IUI vs. single IUI. IUI, intrauterine insemination. 
the initial findings, further highlighting the urgent need for a new welldesigned and sufficiently powered RCT. Until then, results supporting the superiority of double IUI for couples with male factor infertility should be interpreted with great caution.

Although data from a previous meta-analysis have shown that double IUI does not significantly improve pregnancy rates among women with unexplained infertility, ${ }^{15}$ such an approach may have a markedly different outcome among couples with male factor infertility. The rationale for the use of double IUI is that the cumulative number of motile spermatozoa inseminated in double IUI cycles is higher than that in single IUI cycles. Consequently, considering that the clinical pregnancy rate in IUI cycles is strongly associated with the number of motile spermatozoa inseminated, ${ }^{16,17}$ this may be of particular value for couples with male factor infertility. In these couples, the number of spermatozoa inseminated is considerably low compared with couples with other causes of infertility. Thus, because the increase in IUI frequency results in more motile spermatozoa being delivered to the site of fertilisation in each treatment cycle, this may increase pregnancy rates. Furthermore, with double IUI, the time of spermatozoa presence is longer, which may contribute to the increase in the success rate of IUI per cycle. The time of insemination is very important for the success of IUI, ${ }^{18}$ because follicle rupture may occur over a long time interval. With double IUI, a longer interval after hCG administration in $\mathrm{OH} / \mathrm{IUI}$ cycles of living spermatozoa is achieved, which may eventually result in a higher fertilisation rate. ${ }^{19}$

The importance of a technique such as double IUI may be paramount if this technique results in higher pregnancy rates than single IUI because this would have a substantial financial impact in the management of infertile couples. Until recently, IUI was considered the standard method of treatment in cases of unexplained and male factor subfertility. Several studies supported IUI as having the same likelihood of successful pregnancy as IVF while being more cost-effective, with the cost of IUI per pregnancy remaining four to seven times lower than the cost of IVF. ${ }^{6,20,21}$ Nonetheless, despite the availability and ease of performing IUI, other studies support the use of IVF as a first line of therapy based on their findings of higher success rates, shorter times to pregnancy and a trend towards fewer multiple pregnancies. ${ }^{22}$ Furthermore, in a more recent cost-effectiveness analysis, it was calculated that for unexplained and mild male factor subfertility, a full primary IVF cycle is more costeffective than providing IUI followed by IVF. ${ }^{23}$

Consequently, given the controversial evidence described above, it is relatively clear that a treatment modality that could increase pregnancy rates among infertile couples undergoing IUI may indeed further justify its use as a first line treatment. Although initial reports regarding double IUI show promise for couples with male factor infertility, the current available evidence does not have the power or the consistency to support such a shift in clinical practice. Double IUI did not improve pregnancy rates among women with unexplained infertility, ${ }^{15}$ and it is not clear whether double IUI sufficiently improves pregnancy rates in couples with male factor infertility. In addition, we should consider the fact that double IUI increases both the financial cost and burden on the health provider and the couple compared with single IUI. ${ }^{14}$ Consequently, at present, its role is controversial in everyday clinical practice.

Furthermore, we must acknowledge that several limitations are present in our analysis that prevent us from being conclusive. First of all, the quality of the included trials was low, with a high risk of bias according to the Cochrane's risk of bias tool, as shown in Figure 2. Taking into account that the inclusion of trials with high or unclear risk of bias has been previously reported to lead to inflated outcomes in favour of the experimental arm, ${ }^{24}$ we cannot exclude that this may have been the reason for the improved outcomes in favour of double IUI. In addition, we decided not to assess the presence of publication bias through asymmetry tests because of the small number of trials included. Although application of these tests to small numbers of studies is not incorrect, the tests have low statistical power in such cases and may be meaningless. ${ }^{25}$ Therefore, we cannot exclude the presence of such a bias given that publication bias has been previously reported in the field of reproductive medicine. ${ }^{26}$ Many trials that do not favour the experimental arm are left unpublished. Nonetheless, the likelihood of missing trials with negative results is low given that we used the most rigorous search criteria and that we manually searched the abstract books of two major infertility congresses.

However, we cannot overlook the results derived from the available trials. It is clear that even after performing a sensitivity analysis excluding the trial by Liu et al., ${ }^{8}$ a trend in favour of double IUI was still present. Thus, it will be imperative to assess the results of future trials examining the success of double IUI for couples with male factor infertility. An interesting observation that may guide future research is that among the trials included in our analysis, there were differences in the severity of male factor infertility, which may explain the diverse pregnancy rates among eligible trials. For example, whereas Liu et al. ${ }^{8}$ included only patients with mild male factor infertility and found a significant difference between the compared groups, Alborzi et al. ${ }^{12}$ included couples with mild and severe male factor infertility and found no difference between the compared arms. Therefore, future trials may need to include only couples with mild male factor infertility given that the effect of IUI depends on the number of motile spermatozoa that are inseminated. ${ }^{17}$

Another interesting finding related to our analysis was that although three of the studies demonstrated a trend towards better outcomes in couples receiving double IUI, ${ }^{8,9,13}$ two of the studies did not demonstrate any clear benefit. ${ }^{12,14}$ A careful scrutiny of the patients' characteristics shows that these two studies with less favourable results included considerably younger female partners than the other studies (Table 1). Therefore, it may be hypothesized that the requirement for inseminated sperm in the population included in these studies could have been lower than in the studies demonstrating a benefit.

In conclusion, the current systematic review highlights the need for future trials and does not indicate a need to change clinical practice. Despite the fact that receiving two IUIs per treatment cycle was found to result in higher clinical pregnancy rates, the limitations mentioned above prevent us from drawing a definite conclusion. There seems to be a trend towards better outcomes with an increase in the frequency of IUIs per treatment cycle when the cause of infertility is an isolated male factor. However, this must first be validated by large and welldesigned randomized trials.

\section{AUTHOR CONTRIBUTIONS}

NPP thought of the original concept for the study, AZ and NPP performed the literature searches and extracted the data, AZ and NPP performed trial quality assessments, the analyses and wrote the manuscript. $\mathrm{AD}, \mathrm{AG}, \mathrm{CV}$, $\mathrm{EP}$ and GA were involved in the interpretation of the results, and all revised and critically commented on the final draft of the manuscript.

\section{COMPETING FINANCIAL INTERESTS}

The authors declare that they have no competing financial interests.

1 Lipshultz LI, Howards SS, Niederberger CS. Infertility in the Male. 4th ed. Cambridge: Cambridge University Press; 2009. pxi, 677 pp., [8] p. of plates. 
neg

Double intrauterine insemination for male infertility

A Zavos et al

538

2 Willets $\mathrm{AE}$, Combo JM, Brown JN. Clomiphene for the treatment of male infertility. Reprod Sci; e-pub ahead of print November 2012; doi:10.1177/ 1933719112466304.

3 Collins JA, Burrows EA, Wilan AR. The prognosis for live birth among untreated infertile couples. Fertil Steril 1995; 64: 22-8.

4 Tournaye H. Male factor infertility and ART. Asian J Androl 2012; 14: 103-8.

5 Wright VC, Chang J, Jung G, Macaluso M. Assisted reproductive technology surveillance-United States, 2005. MMWR Surveill Sim 2008; 57: 1-23.

6 Goverde AJ, McDonnell J, Vermeiden JP, Schats R, Rutten FF et al. Intrauterine insemination or in-vitro fertilisation in idiopathic subfertility and male subfertility: a randomised trial and cost-effectiveness analysis. Lancet 2000; 355: 13-8.

7 Osuna C, Matorras R, Pijoan JI, Rodriguez-Escudero FJ. One versus two insemination per cycle in intrauterine insemination with sperm from patients' husbands: a systematic review of the literature. Fertil Steril 2004; 82: 17-24.

8 Li W, Gong F, Lu K, Lu G. Comparing the pregnancy rates of one versus two intrauterine insemination (LUIs) in male factor and idiopathic infertility. $J$ Assist Reprod Genet 2006; 23: 75-9.

9 Rani G, Maggioni P, Guermandi E, Testa A, Baron E et al. Efficacy of double intrauterine insemination in controlled ovarian hyperstimulation cycles. Fertil Steril 1999; 72: 619-22.

10 Lu J, loannidis JP, Schmid CH. Quantitative synthesis in systematic reviews. Ann Intern Med 1997; 127: 820-6.

11 Petitti DB. Meta-Analysis, Decision Analysis, and Cost-Effectiveness Analysis: Methods for Quantitative Synthesis in Medicine. Ind ed. New York/Oxford: Oxford University Press; 2000. px, 306 pp.

12 Alborzi S, Motazedian S, Parsanezhad ME, Jannati S. Comparison of the effectiveness of single intrauterine insemination (IUI) versus double IUI per cycle in infertile patients. Fertil Steril 2003; 80: 595-9.

13 Casadei L, Zamaro V, Calcagni M, Ticconi C, Dorrucci M et al. Homologous intrauterine insemination in controlled ovarian hyperstimulation cycles: a comparison among three different regimens. Eur J Obstet Gynecol Reprod Biol 2006; 129: 155-61.

14 Basis T, Haydardedeoglu B, Kilicdag EB, Cot T, Simsek E et al. Single versus double intrauterine insemination in multi-follicular ovarian hyperstimulation cycles: a randomized trial. Hum Reprod 2010; 25: 1684-90.
15 Polyzos NP, Tzioras S, Mauri D, Tatsioni A. Double versus single intrauterine insemination for unexplained infertility: a meta-analysis of randomized trials. Fertil Steril 2010; 94: 1261-6.

16 Campania A, Sakkas D, Stalberg A, Bianchi PG, Comte I et al. Intrauterine insemination: evaluation of the results according to the woman's age, sperm quality, total sperm count per insemination and life table analysis. Hum Reprod 1996; 11: 732-6.

17 Whiner R, Albert M, Dorion A, Bailly M, Berger M et al. Influence of the number of motile spermatozoa inseminated and of their morphology on the success of intrauterine insemination. Hum Reprod 2004; 19: 2060-5.

18 Ghanem ME, Bakre NI, Exam MA, Al Boghdady LA, Helal AS et al. The effects of timing of intrauterine insemination in relation to ovulation and the number of insemination on cycle pregnancy rate in common infertility etiologies. Hum Reprod 2011; 26: 576-83.

19 Testart J, Frydman R. Minimum time lapse between luteinizing hormone surge or human chorionic gonadotropin administration and follicular rupture. Fertil Steril 1982; 37: 50-3.

20 Comhaire F. Economic strategies in modern male subfertility treatment. Hum Reprod 1995; 10(Suppl 1): 103-6.

21 Robinson D, Syrop CH, Hammitt DG. After superovulation-intrauterine insemination fails: the prognosis for treatment by gamete intrafallopian transfer/pronuclear stage transfer. Fertil Steril 1992; 57: 606-12.

22 Chambers GM, Sullivan EA, Shanahan M, Ho MT, Priester $\mathrm{K}$ et al. Is in vitro fertilisation more effective than stimulated intrauterine insemination as a first-line therapy for subfertility? A cohort analysis. Aust NZ J Obstet Gynaecol 2010; 50: 280-8.

23 Pashayan N, Lyratzopoulos G, Mathur R. Cost-effectiveness of primary offer of IVF vs. primary offer of IUI followed by IVF (for IUI failures) in couples with unexplained or mild male factor subfertility. BMC Health Serv Res 2006; 6: 80.

24 Polyzos NP, Polyzos IP, Zavos A, Valachis A, Mari D et al. Obstetric outcomes after treatment of periodontal disease during pregnancy: systematic review and metaanalysis. BMJ 2010; 341: c7017.

25 loannidis JP, Trikalinos TA. The appropriateness of asymmetry tests for publication bias in meta-analyses: a large survey. CMAJ 2007; 176: 1091-6.

26 Polyzos NP, Valachis A, Patavoukas E, Papanikolaou EG, Messinis IE et al. Publication bias in reproductive medicine: from the European Society of Human Reproduction and Embryology annual meeting to publication. Hum Reprod 2011; 26: 1371-6.

Asian Journal of Andrology 